\title{
An approach to design of an intelligent heating system
}

\author{
Valery Stennikov ${ }^{1}$, Evgeny Barakhtenko ${ }^{1}$, and Dmitry Sokolov ${ }^{1, *}$ \\ ${ }^{1}$ Melentiev Energy Systems Institute of Siberian Branch of the Russian Academy of Sciences (ESI SB \\ RAS), Pipeline Energy Systems Department, 130, Lermontov str., Irkutsk, Russia, 664033
}

\begin{abstract}
At present, there is a growing need to solve the design problem of the hydraulic circuit of an intelligent heating system with the required transmission capacity, hydraulic stability and reliability indices. The paper proposes a mathematical statement of the design problem of such a hydraulic circuit and an original methodological approach to solve it by decomposing a general complex problem into several simpler ones (of a smaller dimensionality). The proposed approach makes it possible to tailor the computational procedure to specific features of a heating system to be modeled.
\end{abstract}

\section{Introduction}

Heating systems have a great social and economic significance. Nowadays, they have turned into complex spatially distributed pipeline systems for heat supply to the consumer. The complexity of these systems is related to their closed two-line schemes, multi-ring structure, a variety of heat sources and control elements (pumping and throttling stations, regulators, heat points).

A promising technological platform for future heat supply is intelligent heating systems that integrate the sources, networks and consumers at a new technological level into a single automated system [1]. This will provide these systems with the necessary flexibility under their changing operating conditions, enhance heat supply efficiency, reliability, quality; will help reduce heat losses, smooth out uneven heat consumption, etc.

The available possibility for automatic real-time monitoring and control of operating conditions of all participants involved in the process of heat generation, transmission and consumption will enable a quick response to changes in various parameters in the heating system. This will ensure a two-way mutually coordinated interaction between the consumer and the heating system.

In the context of transition to new concepts for the construction of intelligent heating systems, the growing scale and complexity of these systems, and an increase in the number of active network elements (sensors, pressure and flow regulators, etc.), the demand for mathematical models, methods and algorithms intended to design these systems is increasing.

\footnotetext{
* Corresponding author: $\underline{\text { sokolov dv@isem.irk.ru }}$
} 
To design a heating system, we solve the problem of ensuring the necessary transmission capacity by determining optimal parameters of the system. A common approach to solving this problem is its reduction to the problem of linear programming [24]. The advent of dynamic programming [5] led to a significant development of methods for solving the problems of related to the selection of optimal parameters [6-8]. Some researchers try to use combinatorial methods to solve the problem of optimal design of pipeline systems [9]. In [10], the authors propose the Davidon-Fletcher-Powell method to find a solution. The authors of [11] apply a generalized reduced gradient (GRG) algorithm.

The heuristic algorithms are currently used to develop various approaches to the optimal design of pipeline systems [12-14]. The authors of [15] propose simulated annealing.

In Russia, the method of multi-loop optimization is used to determine the optimal parameters of ring pipeline systems, based on the principle of continuous improvement in solution $[16,8]$. This method is further developed in [17-19]. The authors of [8] propose the method of redundant design schemes to select the configuration of pipeline systems.

Design of pipeline systems involves the methods of hydraulic and thermal calculations, which are considered in [20-24]. In [25-26], the focus is made on the methods for an analysis and improvement of energy system reliability.

At present, to solve the design problems of intelligent heating systems we need to develop:

- a single statement of the design problem of a hydraulic circuit of an intelligent heating system with required transmission capacity and indices of hydraulic stability and reliability; - a single methodological approach to solve the design problem of the hydraulic circuit of an intelligent heating system.

\section{Mathematical statement of the problem}

The statement of the design problem of the hydraulic circuit of intelligent heating system has the following form: a specified network scheme is a calculated (design) scheme that consists of $m$ nodes and $n$ branches, and is represented by a directed graph $G_{\mathrm{HN}}=(J, I)$, where $J=J_{\mathrm{C}} \cup J_{\mathrm{S}} \cup J_{\mathrm{B}}$ - a set of vertices (nodes) that includes a set of consumers $J_{\mathrm{C}}$, sources $J_{\mathrm{S}}$ and branching points on the scheme $J_{\mathrm{B}} ; I=I_{\mathrm{P}} \cup I_{\mathrm{S}} \cup I_{\mathrm{C}}$, where $I_{\mathrm{P}}=I_{\mathrm{N}} \cup I_{\mathrm{E}}$ - a set of branches of linear (pipeline) part of the network that consists of a set of existing $I_{\mathrm{C}}$ and new $I_{\mathrm{N}}$ branches; $I_{\mathrm{C}}$ and $I_{\mathrm{S}}$ - sets of consumer branches and source branches, respectively; $I_{\mathrm{PS}} \subset I$ - branches in which the pumping stations are either installed or can be installed. The lengths $L_{i}(i \in I)$ for all the branches are specified.

When solving the problem, we should minimize the total cost function of the heating system, that has the form:

$$
\begin{aligned}
& F(\mathbf{d}, \mathbf{H}, \mathbf{x}, \mathbf{P})=\sum_{i \in I_{\mathrm{P}}} F_{i}^{\mathrm{P}}\left(d_{i}\right)+\sum_{i \in I_{\mathrm{PS}}} F_{i}^{\mathrm{PS}}\left(H_{i}, x_{i}\right)+ \\
& +\sum_{\substack{i \in I \\
\varphi(i)=(j, k)}} F_{i}^{\mathrm{E}}\left(x_{i}, d_{i}, P_{j}, P_{k}\right)+\sum_{j \in J_{\mathrm{C}}} F_{i}^{\mathrm{C}}\left(P_{j}\right) \rightarrow \min ,
\end{aligned}
$$

where $F_{i}^{\mathrm{P}}$ - the costs of pipeline construction and operation; $F_{i}^{\mathrm{PS}}-$ costs of pumping station construction and operation; $F_{i}^{\mathrm{E}}$ and $F_{j}^{\mathrm{C}}$ - costs of electricity used to pump the transported medium and the costs of its supply to the consumer, respectively ; d $-n$-dimensional vector of pipeline diameters; $\mathbf{H}-n$-dimensional vector of heads at pumping stations; $\mathbf{x}-$ $n$-dimensional vector of transported medium flow rates in the network branches; $\mathbf{P}-m$ - 
dimensional vector of pressure at network nodes; $\varphi(i)$ - function putting each branch $i \in I$ in correspondence with a couple of incident nodes $(j, k)$.

The model of flow distribution in a pipeline network has the following form:

$$
\begin{gathered}
\mathbf{A x}=\mathbf{G}, \\
\mathbf{A}^{\mathrm{T}} \mathbf{P}+\mathbf{H}=\mathbf{f}(\mathbf{s}, \mathbf{x}),
\end{gathered}
$$

where $\mathbf{A}-$ an $(m \times n)$-matrix of incidence of the calculated scheme; $\mathbf{G}-m$-dimensional vector of nodal sinks and sources of the transported medium; $\mathbf{f}-n$-dimensional vector function with elements $f_{i}\left(s_{i}\left(d_{i}\right), x_{i}\right)=s_{i}\left(d_{i}\right) x_{i}\left|x_{i}\right|^{\beta-1}, i \in I$, that reflect the law of pressure drop in the network branch, $s_{i}\left(d_{i}\right)$-hydraulic resistance of pipeline.

A system of constraints consists of the following components:

$$
\begin{gathered}
P_{j}^{\min } \leq P_{j} \leq P_{j}^{\max }, \quad j \in J ; \\
d_{i} \in D, \quad i \in I_{\mathrm{P}} ; \\
H_{i} \in H_{\mathrm{S}}, \quad i \in I_{\mathrm{Ps}} ; \\
v_{i}^{\min } \leq v_{i}\left(x_{i}, d_{i}\right) \leq v_{i}^{\max }, \quad i \in I_{\mathrm{P}} ;
\end{gathered}
$$

where $P_{j}^{\min }$ and $P_{j}^{\max }$ - lower and upper pressure constraints; $D$ - a set of diameters of standard pipelines; $v_{i}^{\min }$ and $v_{i}^{\max }$ - lower and upper constraints on the transported medium velocity $v_{i} ; H_{\mathrm{S}}-$ a set of heads at pumping stations that can be installed.

The reliability requirements impose the constraints that are determined by standard values of:

- availability factor at all consumer nodes

$$
K_{j} \geq K^{\mathrm{min}}, \quad j \in J_{\mathrm{C}}
$$

- failure-free operation probability index for all consumer nodes

$$
R_{j} \geq R^{\min }, \quad j \in J_{\mathrm{C}}
$$

where $K^{\min }$ and $R^{\min }$ are lower constraints on the availability factor and probability of failure-free operation, respectively.

Constraints on the required level of hydraulic stability for each consumer are

$$
\omega_{i}\left(x_{i}, G_{j}, G_{k}\right) \geq \omega_{i}^{\min }, \quad i \in I_{\mathrm{C}}, \quad \varphi(i)=(j, k),
$$

where $\omega_{i}$ - function of hydraulic stability of the consumer, $\omega_{i}^{\min }$ - required value of a coefficient of hydraulic stability of the consumer.

By solving the problem of function minimization (1), subject to (2)-(10), we should determine the parameters of the intelligent heating system.

\section{A proposed approach to solve the problem}

We propose an original methodological approach to solve the stated design problem of the hydraulic circuit of the intelligent heating system. The approach is based on the concept of decomposition of a general complex problem into a set of less complex problems. By decomposing the general problem we obtain the following conceptual problems to solve:

- select the network configuration;

- determine optimal parameters of the hydraulic circuit;

- estimate and ensure the necessary level of system reliability;

- carry out hydraulic and thermal calculations.

The problem of selection of the network configuration lies in determining the best graph of the system. It is solved by the method of redundant design scheme [9]. The 
determination of optimal hydraulic circuit parameters means the search for a solution ensuring the best transmission capacity of the network by replacing pipelines and installing (retrofitting) pumping stations. This problem is solved by the methods of dynamic programming and multi-loop optimization $[17,18]$. The problems of hydraulic and thermal calculations are reduced to solving systems of linear and nonlinear equations. These problems are solved by the effective methods developed at Melentiev Energy Systems Institute of SB RAS [8, 23, 24].

The proposed methodological approach suggests the following steps to solve the design problem of hydraulic circuit of intelligent heating system (Fig.1):

Step 1. Build a redundant design scheme of a heating system.

Step 2. In the case that there is no need to solve the problem of network configuration selection, go to step 4.

Step 3. Solve the problem of network configuration selection.

Step 4. Determine optimal parameters of the hydraulic circuit.

Step 5. Calculate and assess reliability indices.

Step 6. In the case that the required reliability level is provided, go to step 8.

Step 7. Solve the problem dealing with addition of backup ties to provide the required reliability level. If these ties are necessary - go to step 1, if not - go to step 3 .

Step 8. Calculate and analyze hydraulic and thermal conditions.

Step 9. Based on the analysis of hydraulic and thermal conditions make a decision on the correction of the parameters. If the correction is necessary, go to step 4.

Step 10. Calculate and analyze the cost-effectiveness indices.

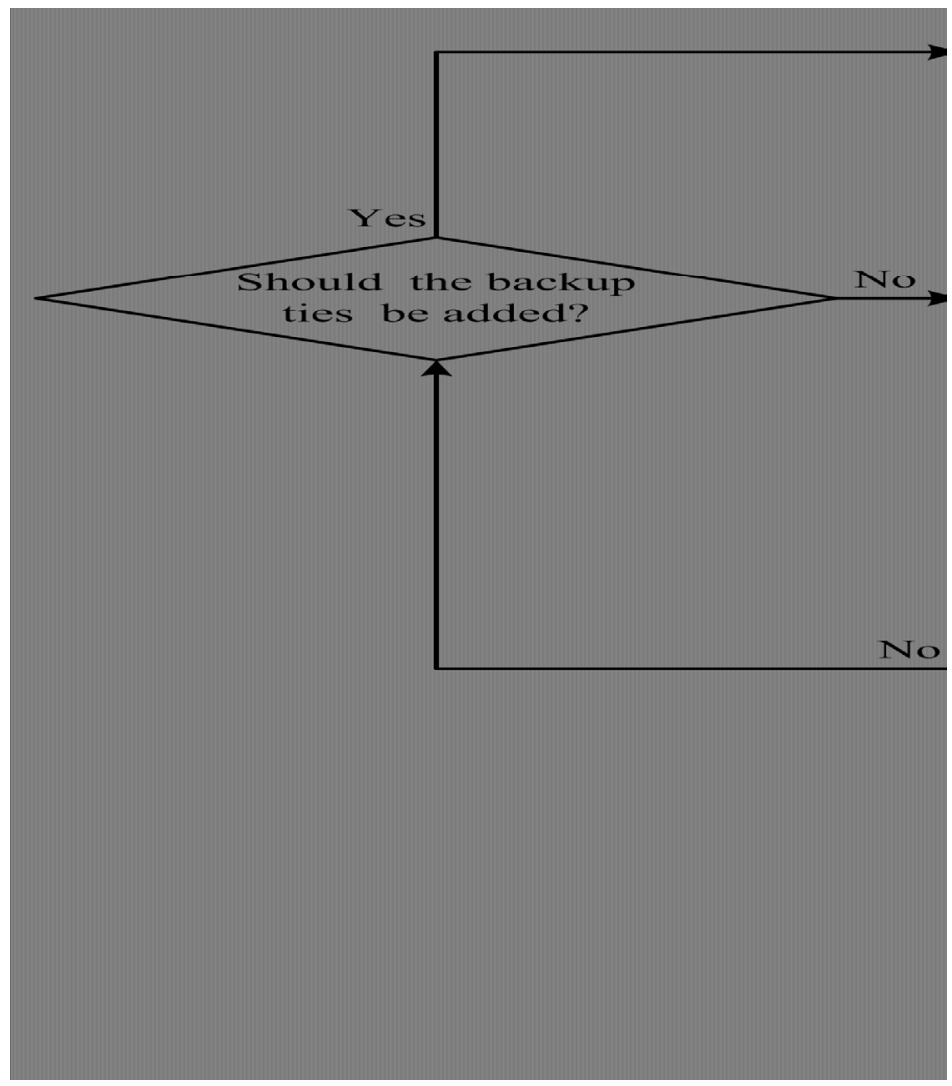

Fig. 1. A proposed algorithm. 
The developed methodology was applied to investigate the possibility of construction of an intelligent heating system in the district Novo-Lenino of the city of Irkutsk, which is a typical urban district with prevailing residential housing. The solutions obtained from the calculation are intended to develop this system and transform it into an intelligent system to provide better comfort, quality, and reliability of heat supply to consumers in this district.

\section{Conclusion}

We propose a mathematical statement of the design problem of hydraulic circuit of intelligent heating system with the required transmission capacity and necessary indices of hydraulic stability and reliability. An original methodological approach is proposed to solve the formulated problem. This approach suggests decomposition of a general complex problem into several less complex problems (of smaller dimensionality and complexity).

The proposed methodological approach makes it possible to:

- Fully consider the specific characteristics of equipment applied, ways of its installation and operation;

- Use complex nonlinear mathematical models;

- Adjust computational procedure to specific features of a system to be modeled.

The developed methodology can be used to make recommendations to improve and transform complex heating systems into intelligent systems, enhance their efficiency and quality of heat supply to consumers.

The research was supported by Russian Science Foundation (grant No. 17-19-01209).

\section{References}

1. H. Lund, S. Werner, R. Wiltshire, S. Svendsen, J. E. Thorsen, F. Hvelplund, Energy, 68, 1-11 (2014)

2. E. Alperovits, U. Shamir, Water Resour. Res. 13, 885-900 (1977)

3. I. Goulter, J. Water. Res. Plan. Manage. Div. Am. Soc. Civ. Eng., 118, 238-248 (1992)

4. G. Eiger, U. Shamir, A. Ben-Tal, Water Resour. Res. 30, 2637-2646 (1994)

5. R. E. Bellman, Dynamic programming (1957)

6. J. R. Appleyard, Build. Serv. Eng. 45, 191-204 (1978)

7. L. Garbai, L. Mobnar, Colloq. math, societatic Janos Bolyai. 12, 373-390 (1974)

8. A. Merenkov, V. Khasilev, The Theory of Hydraulic Circuits (1985)

9. J. Gessler, Proc. of the Ninth International symposium on Urban Hydrology, Hydraulics and Sediment Control, (1982)

10. A. Chiplunkar, S. Mehndiratta, P. Khanna, J. Env. Eng. Div. Am. Soc. Civ. Eng., 112, 264-279 (1986)

11. K. Lansey, L. Mays, J. Hydraul. Div. Am. Soc. Civ. Eng. 115, 1401-1418 (1989)

12. K. McClymont, E. Keedwell, D. Savic, Environ Modell Softw, 69, 414-424 (2015)

13. D. Savic, G. Walters, J. Water Resour. Plann. Manag. 123, 67-77 (1997)

14. J. W. Nicklow, P. M. Reed, D. Savic, T. Dessalegne, L. Harrell, A. Chan-Hilton, M. Karamouz, B. Minsker, A. Ostfeld, A. Singh, E. Zechman, J. Water Res. Plan. Man. 136 (4), 412-432 (2010)

15. M. Cunha, J. Sousa, J. Water Res. Plan. Manage. Div. Soc. Civ. Eng., 125, 215-221 (1999) 
16. S. Sumarokov, Ekonomika i matematicheskie metody, 12, 1016-1018 (1976)

17. V. Stennikov, E. Barakhtenko, D. Sokolov, T. Oshchepkova, Sustaining Power Resources through Energy Optimization and Engineering Premier Reference Source, 76-101 (2016)

18. V. Stennikov, E. Barakhtenko, D. Sokolov, Therm Eng, 64, 518-525 (2017)

19. D. Sokolov, V. Stennikov, T. Oshchepkova, E. Barakhtenko, Therm Eng, 59, 337-343 (2012)

20. D. W. Martin, G. Peters, IWES J., 1963

21. U. Shamir, C. D. D. Howard, J. Hydraul. Div. ASCE, 94 (1968)

22. E. Todini, S. Pilati, System Analysis and Simulation, 1-20 (1987)

23. N. Novitskii, Therm Eng, 60, 1051-1060 (2013)

24. V. Tokarev, Z. Shalaginova, Therm Eng, 63, 68-77 (2016)

25. M. Haghifam, M. Manbachi, Int J Elec Power, 33, 385-393 (2011)

26. I. Postnikov, V. Stennikov, E. Mednikova, A. Penkovskii, Energy Procedia, 105, 30833088 (2017) 